\title{
Peripheral Osteoma: Unusual Presentation in the Mandibular
}

\section{Notch}

\begin{abstract}
Keywords: Peripheral osteoma; Sigmoid notch; Temporo-
\end{abstract} mandibular joint; Oral \& Maxillofacial Surgery

\begin{abstract}
This case report demonstrates an unusual presentation of a large peripheral osteoma affecting the left mandibular notch of a 42-year old female. Only five other cases involving this region have ever been reported in the English literature.

The patient complained of a constant 'contraction' like movement of her jaw over the course of one-year, with associated pre-auricular pain in the left temporo-mandibular joint region. Orthopantomographic investigation revealed a radiopaque mass related to the left mandibular notch with a subsequent cone beam computed tomography reporting a large pedunculated bony mass with a lobulated outline, extending into the infra temporal fossa.

A working diagnosis of peripheral osteoma was reached, and surgery suggested to prevent further growth and to relieve symptoms. The patient declined treatment, and was therefore offered periodic clinical and radiographic follow up.
\end{abstract}

\section{Introduction}

Osteomas are benign osteogenic neoplasms characterized by proliferation of compact or cancellous bone. They can be central, peripheral, or extra skeletal. Peripheral osteomas of the jawbones are uncommon and lesions involving the mandibular notch are extremely rare [1-3]. Craniofacial lesions occur most frequently in the paranasal sinuses (frontal $>$ ethmoidal $>$ maxillary) [4].

The aetiology of these lesions is unknown, however most researchers consider neoplastic, developmental and reactive causes [5]. Osteomas are more common in young adults and there is no sex predilection. Peripheral osteoma usually appears as a unilateral, sessile or pedunculated, well-circumscribed, mushroom-like mass. Symptoms may include asymmetry, interference with oral function and malocclusion, however, lesions are generally asymptomatic [1].

Patients with multiple jaw osteomas should be investigated for Gardener's syndrome whereby osteomas occur in combination with polyposis coli and multiple fibrous tumours/sebaceous cysts of the skin. It is important to identify this condition early as extra-intestinal lesions may present before multiple colonic polyps (adenomatous polyposis coli), which will typically undergo malignant transformation by the fourth decade. Family members should also be investigated, as the condition is hereditary [6].

\section{Case Presentation}

A 42-year-old female was referred to the Oral and Maxillofacial surgery clinic, complaining of a constant 'contraction' like movement of her jaw over a one-year period. She was also experiencing preauricular pain in the left temporo-mandibular joint (TMJ) region.

\section{Journal of}

Clinical \& Medical Case Reports

Daniel Palermo ${ }^{1 *}$, Anne Begley ${ }^{2}$ and Paul Nixon ${ }^{2}$

'Alder Hey Children's Hospital, East Prescot Road, Liverpool, L14 $5 A B, U K$

${ }^{2}$ Liverpool University Dental Hospital, Pembroke Place, Liverpool, Merseyside, L3 5PS, UK

Address for Correspondence:

Daniel Palermo, Alder Hey Children's Hospital, East Prescot Road, Liverpool, L14 5AB, UK, Tel: 0151228 4811; E-mail: Daniel.Palermo@alderhey.nhs.uk

Submission: 15 April, 2016

Accepted: 13 May, 2016

Published: 18 May, 2016

Copyright: (๑) 2016 Palermo D, et al. This is an open access article distributed under the Creative Commons Attribution License, which permits unrestricted use, distribution, and reproduction in any medium, provided the original work is properly cited.

She did not report any previous trauma.

Clinical examination revealed no swelling or facial asymmetry therefore a full Orthopantomograph (OPG) radiograph was requested to assess for likely sources of the patient's presenting complaints, which were expected to be a TMJ related disorder or dental pathology. However the radiograph revealed a radiopaque mass related to the left mandibular notch, which was not palpable clinically (Figure 1). A cone beam computed tomography (CBCT) scan reported an 18 x $13 \mathrm{x}$ $14 \mathrm{~mm}$ bony mass arising from the left mandibular notch (Figure 2). The lesion had a pedunculated shape with a lobulated outline (Figure $3)$. The appearance of dense bone suggested an osteoma.

Due to the radiographic appearance and peripheral nature of the lesion, the two main differential diagnoses established were a peripheral osteoma or an exostosis. Both lesions have similar histological appearances and therefore need to be differentiated clinically. Unlike the lesion in this case, exostoses are not usually solitary. In addition, lesions stop growing after puberty and are commonly related to areas of attached gingivae.

By contrast, peripheral osteomas present as solitary, usually asymptomatic masses of long duration. Radiographically lesions are well circumscribed, oval and have a similar density to the surrounding

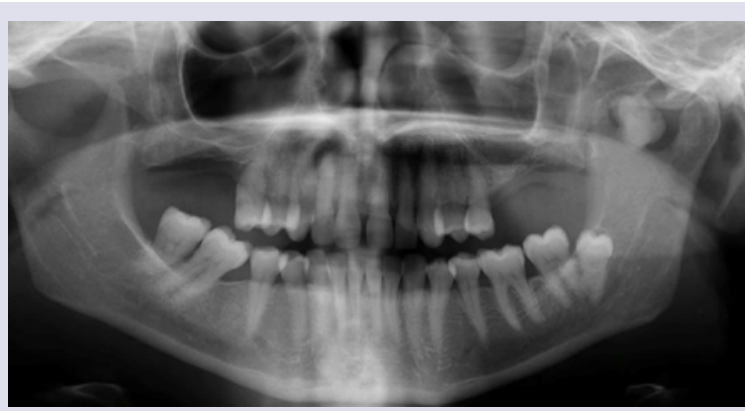

Figure 1: A panoramic radiograph showing a well-defined, radiopaque mass related to the left mandibular notch. 

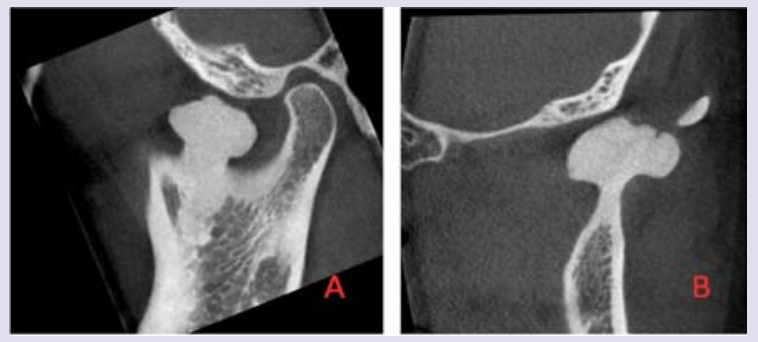

Figure 2: coronal views.

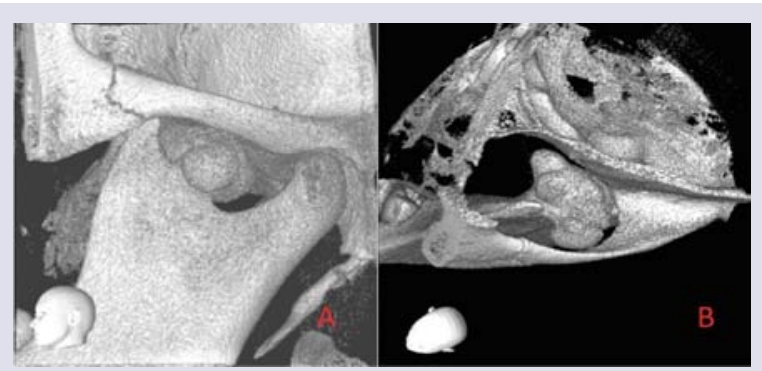

Figure 3: Three-dimensional CBCT images of the lesion in A. lateral and $\mathrm{B}$. superior views.

bone. Histological investigation will be needed to confirm the type of osteoma present i.e. compact, cancellous, or mixed. However, peduncluated osteomas are usually cancellous in nature [5].

A working diagnosis of peripheral osteoma of the left mandibular notch of the mandible was reached. Surgery was suggested to prevent further symptoms and growth.

Appropriate treatment options were complete surgical removal of the lesion (at the cortical bone base) or periodic clinical and radiographic follow up. Surgery is not generally necessary, unless the lesion is symptomatic or actively growing.

In the mandible, intraoral approaches are preferable for cosmetic reasons. There are no reports of osteomas undergoing malignant change and recurrence is very rare. Although surgery was indicated, the patient declined treatment, and was therefore offered periodic clinical and radiographic follow up.

\section{Discussion}

To our knowledge, only 5 cases of peripheral osteoma involving the mandibular notch have ever been reported in the English literature [2,3,7-9]. While peripheral osteomas of the mandible are uncommon, it is extremely rare in the mandibular notch. Johann et al. reported that only $1.6 \%$ of all peripheral osteomas of the mandible are found in the mandibular notch [1].

Cases of an osteoma related to the mandibular notch (including this case) have involved four females and two males, with a large age range from 16 to 78 years of age. Four cases have originated from the right side, and two from the left. Sizes ranged from $18 \times 13 \times 14$ $\mathrm{mm}$ to $36 \times 35 \times 30 \mathrm{~mm}$ and four of the six cases were symptomatic. Previous symptoms have involved progressive swelling and maxillary pain. Only one patient reported a history of traumatic injury [9].

In the cases treated surgically, subsequent histological examination revealed mature compact bone with wide irregularly distributed trabecula, copious fibrovascular channels, and infrequent osteoblasts [2,9].

Exostoses are histologically similar to osteomas and must be excluded as above. Exostoses are localised, benign, peripheral outgrowths of bone. They are often found on long bones related to areas of muscle attachment, however in the oral cavity they are commonly found as torus palatinus (found in the midline of the hard palate), torus mandibularis (related to the lingual aspect of the mandible), and buccal bone exostoses (occurring buccally to the premolar and molar regions of the maxilla and mandible) [10].

The cause of exostoses is unknown, however some authors suggest that a functional adaption to occlusal trauma is likely. Treatment is rarely required but may be considered if lesions are traumatized or interfere with oral hygiene practices, denture provision, periodontal therapy, or speech/tongue space. Bone harvested from exostosis removal can be utilised as an autogenous graft for the treatment of alveolar defects or for periodontal/implant surgery [10].

In summary, this case highlights a rare differential diagnosis to consider for patients who present with jaw ache. Despite the large size of the lesion in a very compact region, the patient complained of relatively mild symptoms. The lesion was also very difficult to palpate and to fully appreciate on an OPG radiograph, which demonstrates the benefits of $\mathrm{CBCT}$ in imaging such lesions.

\section{References}

1. Johann AC, de Freitas JB, de Aguiar MC, de Araujo NS, Mesquita RA (2005) Peripheral osteoma of the mandible: case report and review of the literature. J Craniomaxillofac Surg 33: 276-281.

2. Rao S, Roa S, Pramod DS (2015) Transoral removal of peripheral osteoma at sigmoid notch of the mandible. J Maxillofac Oral Surg 14(Suppl 1): S255-S257.

3. Iwai T, Izumi T, Baba j, Maegawa J, Mitsudo K, et al. (2013) Periphera osteoma of the mandibular notch: report of a case. Iran J Radiol 10: 74-76.

4. Woldenburg Y, Nash M, Bodner L (2005) Peripheral osteoma of the maxillofacial region. Diagnosis and management: a study of 14 cases. Med Oral Patol Oral Cir Bucal 10 Suppl 2: E139-E142.

5. Sayan NB, Ucok C, Karasu HA, Gunhau O (2002) Peripheral osteoma of the oral and maxillofacial region: a study of 35 new cases. J Oral Maxillofac Surg 60: 1299-1301.

6. Basaran G, Erkan M (2008) One of the rarest syndromes in dentistry: Gardener syndrome. Eur J Dent 2: 208-212.

7. Sekerci AE, Sahman H, Ertas ET, Sisman $Y$ (2011) An unusual peripheral osteoma of the mandibular notch: a case report with computed tomography evaluation. Oral Radiol 27: 78-82.

8. Schulze D (2008) Radiographic diagnostics: Osteoma of the left mandibular notch. Quintessence Int 39: 702-703.

9. Bessho K, Murakami KI, lizuka T, Ono T (1987) Osteoma in mandibular condyle. Int J Oral Maxillofac Surg 16: 372-375.

10. Smitha K, Smitha GP (2015) Alveolar exostosis - revisited: A narrative review of the literature. Saudi J Dent Res 6: 67-72. 